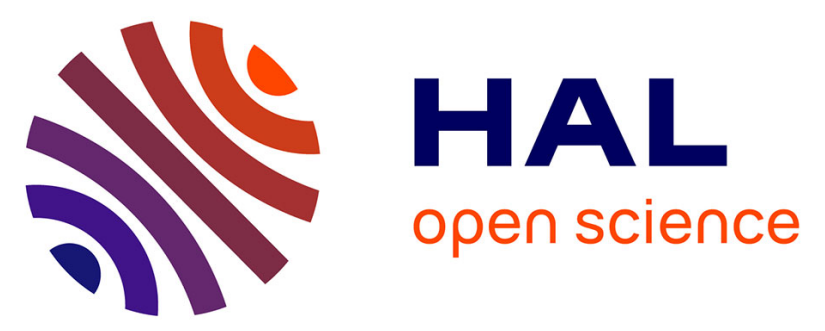

\title{
Propriétés mécaniques en fatigue à grands nombres de cycles des composites carbone époxy
}

Laurent Gornet, Ophélie Westphal, Modesto Mateos, Alina Krasnobrizha, Patrick Rozycki, Catherine Peyrac, Fabien Lefebvre

\section{- To cite this version:}

Laurent Gornet, Ophélie Westphal, Modesto Mateos, Alina Krasnobrizha, Patrick Rozycki, et al.. Propriétés mécaniques en fatigue à grands nombres de cycles des composites carbone époxy : Détermination de la limite de fatigue par la méthode d'auto-échauffement. Revue des composites et des matériaux avancés $=$ Journal of Composite and Advanced Materials, 2015, 25 (2), pp.181-200. 10.3166/rcma.25.181-200 . hal-01240283

\section{HAL Id: hal-01240283 \\ https://hal.science/hal-01240283}

Submitted on 26 May 2017

HAL is a multi-disciplinary open access archive for the deposit and dissemination of scientific research documents, whether they are published or not. The documents may come from teaching and research institutions in France or abroad, or from public or private research centers.
L'archive ouverte pluridisciplinaire HAL, est destinée au dépôt et à la diffusion de documents scientifiques de niveau recherche, publiés ou non, émanant des établissements d'enseignement et de recherche français ou étrangers, des laboratoires publics ou privés. 


\title{
Propriétés mécaniques en fatigue à grands nombres de cycles des composites carbone époxy
}

\author{
Détermination de la limite de fatigue \\ par la méthode d'auto-échauffement
}

\section{Laurent Gornet $^{1}$, Ophélie Westphal ${ }^{1,2}$, Modesto Mateos ${ }^{1}$, Alina Krasnobrizha ${ }^{1}$, Patrick Rozycki ${ }^{1}$, Catherine Peyrac ${ }^{2}$, Fabien Lefebvre ${ }^{2}$}
1. École Centrale de Nantes, Institut de recherche en Génie Civil et Mécanique GeM, 1 Rue de la Noё, BP 92101, 44321 Nantes Cedex 3, France laurent.gornet@ec-nantes.fr,patrick.rozycki@ec-nantes.fr

2. Compinnov Openlab, Cetim, 52, avenue Félix Louat-60304, Senlis Cedex ophelie.westphal@ec-nantes.fr,fabien.lefebvre@cetim.fr

\begin{abstract}
RESUME. Ce travail concerne la détermination des propriétés mécaniques en fatigue d'un pli unidirectionnel carbone époxy TR50-R367-2. Les caractéristiques mécaniques de fatigue à grand nombre de cycles de ce matériau sont déterminées en quelques heures à l'aide de la méthode d'auto-échauffement. Les propriétés de fatigue obtenues par cette méthode innovante sont validées à l'aide de la construction complète de la courbe de Wöhler réalisée en quelques mois. La méthode proposée permet donc de déterminer la limite de fatigue d'un carbone époxy en quelques heures contrairement aux essais classiques de fatigue. Enfin la simulation des dissipations est réalisée à l'aide d'un modèle élastoplastique endommageable à dérivées fractionnaires. Cette approche permet de reproduire les boucles d'hystérésis et de simuler l'évolution de la température de l'éprouvette.
\end{abstract}

ABSTRACT. This work deals with the fatigue mechanical properties determination of a unidirectional TR50-R367-2 carbon fibre reinforced epoxy resin. The high-cycle fatigue mechanical characteristics of this material are determined in only several hours by using the self-heating test method. The fatigue properties obtained with this innovative method are validated by comparison with Wöhler curves constructed from several months lasting tests. Contrary to fatigue tests, the proposed method enables to determine the endurance limit of a carbon fibre reinforced composite in only several hours. Finally, the dissipation simulation is fulfilled by using a damage elastoplastic fractional derivative model. This approach enables on the one hand to reproduce the hysteresis loops and on the other hand to simulate the sample temperature. 
MotS-CLES: dérivée fractionnaire, essai auto-échauffement, carbone thermoplastique, fatigue.

KEYWORDS: fractional derivative, self-heating test, fatigue, carbon, thermoset.

\section{Extended abstract}

The rapid determination of high-cycle fatigue properties by the self-heating test method enables to obtain the endurance limit of a composite elementary ply in only several hours. This method, which was initially developed for metallic materials by means of dissipation analysis (Luong et Dang Van 92, Chrysochoss et Louche 2000, G. La Rosa et Risitano 2000) has been adapted by the authors for carbon fibre reinforced epoxy resin and carbon fibre reinforced thermoplastic composite materials (Westphal 2014, Gornet et al. 2015). The method is presented for a high strength unidirectional TR50-R367-2 carbon fibre reinforced epoxy resin, which enables to determine the fatigue limit of the material elementary ply.

The self-heating method consists in subjecting the specimen in an accelerated way to a degradation state equivalent to the one which would have been obtained during a fatigue test near its endurance limit. The mechanical tests are fulfilled under a canonical basis of laminates in order to identify the quasi-static and fatigue behaviour for the carbon fibre reinforced epoxy resin elementary ply. An elastoplastic damage fractional derivative model is developed to simulate the mechanical behaviour of the material elementary ply by proposing a mechanical dissipation in accordance with the temperature increase measured during the selfheating tests. The model identification is presented for the woven HexForce 43199/SR8100 carbon fiber epoxy matrix material. The self-heating tests enable to estimate the endurance limits of the composite elementary ply for the directions dominated by matrix behaviour. Furthermore, it is experimentally demonstrated that the fatigue canonical basis stratifications number may be limited by only using a quasi-isotropic sample instead of angle plies $+/-45$ and cross plies $0 / 90$. The selfheating tests have been performed at room temperature in traction/traction with $5 \mathrm{~Hz}$ frequencies with an apparent average stress or by imposing an $\mathrm{R}$ stress ratio. The self-heating method exhibits a low sensitivity to the samples imperfections and the results are therefore equivalent to the average values obtained from fatigue traditional tests. The finite element simulation of the thermal exchanges during the self-heating tests has enabled to better understand the affecting dissipative mechanisms. Finally, a simplified Wöhler curve construction method is proposed by combining the traditional low cycle fatigue tests approach with the results from the self-heating test method that enables to determine the fatigue limit.

\section{Introduction}

La détermination rapide des propriétés de fatigue à grand nombre de cycles par la méthode d'auto-échauffement permet d'obtenir la limite d'endurance du pli 
élémentaire d'un composite en quelques heures. Cette méthode initialement développée pour les matériaux métalliques à partir de l'étude des dissipations (Luong et Dang Van, 1992 ; Chrysochoss et Louche 2000 ; La Rosa et Risitano, 2000) a été adaptée par nos soins pour des matériaux composites stratifiés carboneépoxy et carbone-thermoplastiques (Westphal, 2014 ; Gornet et al., 2015). La méthode est présentée dans le cas du comportement d'un pli unidirectionnel de carbone époxy haute résistance TR50 R367-2. Cette méthode permet de déterminer la limite de fatigue du pli élémentaire de matériau.

La méthode d'auto-échauffement consiste à amener de manière accélérée une éprouvette dans un état de dégradation équivalent à celui qu'elle aurait obtenu lors d'un essai de fatigue proche de sa limite d'endurance. Les essais mécaniques sont menés sur une base canonique d'éprouvettes afin d'identifier le comportement quasi statique et de fatigue du pli élémentaire de carbone époxy. Un modèle de comportement élastoplastique endommageable à dérivées fractionnaires est proposé afin de simuler le comportement mécanique du pli de carbone époxy tout en proposant une dissipation mécanique en accord avec les élévations de température mesurées lors des essais d'auto-échauffements. L'identification du modèle est présentée pour le tissu carbone époxy équilibré HexForce 43199/SR8100. Les essais d'auto-échauffement ont permis d'estimer les limites d'endurance du pli élémentaire de composite pour les directions dominées par le comportement de la matrice. De plus il est prouvé expérimentalement que l'on peut limiter le nombre de stratifications de la base canonique de fatigue en utilisant uniquement une éprouvette quasi isotrope. Les essais d'auto-échauffement ont été réalisés à température ambiante en traction/traction à une fréquence de $5 \mathrm{~Hz}$ pour une contrainte moyenne apparente sur le stratifié ou pour un chargement à contrainte moyenne imposée. La méthode d'auto-échauffement est peu sensible aux imperfections de l'éprouvette et les résultats obtenus sont donc assimilables à ceux de la valeur moyenne des essais traditionnels de fatigue. La simulation éléments finis des échanges thermiques lors des essais d'auto-échauffement a permis de mieux comprendre les mécanismes dissipatifs mis en jeu. Enfin nous proposons de construire une courbe de Wöhler simplifiée en combinant l'approche traditionnelle des essais de fatigue à faible nombre de cycles complétée par les résultats issus de la méthode d'auto échauffement permettant de déterminer la limite de fatigue.

\section{Modélisation des composites stratifiés}

La modélisation des matériaux composites est effectuée à l'échelle intermédiaire appelée méso-échelle qui a été utilisée avec succès sur différents matériaux depuis plus de vingt ans (Ladevèze et Le Dantec, 1992 ; Allix et al., 1994, Ladevèze et al., 1998 ; Marguet et al., 2007). Dans le cas des stratifiés, le composite est décrit à l'échelle méso comme un empilement de monocouches homogènes dans l'épaisseur et d'interfaces interlaminaires (figure 1). L'interface interlaminaire est introduite pour modéliser les phénomènes de délaminage. 


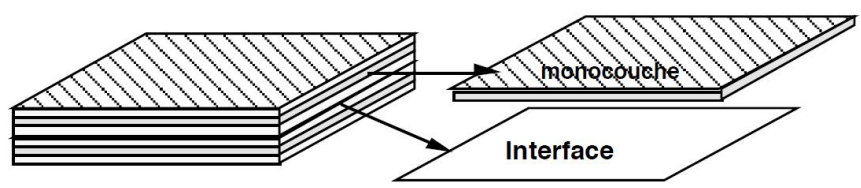

Figure 1. Modélisation du stratifié : couches et interfaces

Un modèle de délaminage a été récemment développé pour les sollicitations de fatigue (Gornet et Ijaz, 2011). Une version non locale est proposée dans (Ijaz et al., 2014). Une fois déterminé le comportement mécanique de ses constituants élémentaires, le comportement de n'importe quel stratifié est établi à partir des propriétés de ses seuls constituants. Le comportement loin des bords du stratifié est obtenu à l'aide de la théorie des plaques stratifiées. Les composites carbone époxy sont notamment utilisés dans la réalisation de voiliers de courses océaniques entièrement réalisés en structures sandwiches (Gornet et al., 2006). Le comportement en cisaillement plan issu d'un essai de traction réalisé sur une éprouvette tissée HexForce 43199/SR8100 en traction [+45/-45]2s. La simulation est menée avec le modèle élastoplastique endommageable (Ladevèze et Le Dantec, 1992). Ce modèle a été modifié en cisaillement plan afin de prendre en compte les boucles d'hystérésis et les effets de vitesses de chargement à l'aide d'une dérivée fractionnaire. Le modèle proposé permet de simuler la dissipation de la boucle d'hystérésis responsable de l'échauffement de l'éprouvette pendant l'essai d'autoéchauffement. Cet essai est destiné à la caractérisation des propriétés de fatigue à grand nombre de cycles. L'énergie de déformation en contraintes planes du pli élémentaire est rappelée ci-après :

$$
\begin{aligned}
& E_{D}=\frac{1}{2\left(1-d_{1}\right)}\left[\frac{\phi_{1}\left(\left\langle-\sigma_{11}\right\rangle_{+}\right)}{E_{1}^{0}}+\frac{\phi_{2}\left(\left\langle\sigma_{11}\right\rangle_{+}\right)}{E_{1}^{0}}+\frac{\left\langle-\sigma_{22}\right\rangle_{+}^{2}}{E_{2}^{0}}+\frac{\left\langle\sigma_{22}\right\rangle_{+}^{2}}{E_{2}^{0}\left(1-d_{2}\right)}\right] \\
& +\frac{1}{2\left(1-d_{1}\right)}\left[-\frac{2 v_{12}^{0}}{E_{1}^{0}} \sigma_{11} \sigma_{22}+\frac{\sigma_{12}^{2}}{G_{12}^{0}\left(1-d_{12}\right)}\right]
\end{aligned}
$$

Les variables d'endommagement $d_{2}$ et $d_{12}$ affectent respectivement le module transverse $E_{2}^{0}$ et le module de cisaillement plan $G_{12}^{0}$. Ces variables sont associées aux phénomènes de microfissuration de la matrice et à la détérioration de la liaison fibre matrice. La rupture des fibres de type élastique fragile est gouvernée quant à elle par la variable d'endommagement $d_{1}$. Les fonctions $\phi_{1}$ et $\phi_{2}$ permettent de prendre en compte la non-linéarité observée en compression ou en traction. Il faut noter que ce dernier phénomène est de plus en plus rencontré sur les composites unidirectionnels à fibre longue de carbone et résine époxy. Cette non-linéarité peut être attribuée à l'ondulation des fibres. La fonction $\phi_{2}$ est choisie de la forme : 


$$
\phi_{2}\left(\left\langle-\sigma_{11}\right\rangle_{+}\right)=-\frac{\left.2 \mid \gamma_{t}\left\langle\sigma_{11}\right\rangle_{+}+\ln \left(1+\gamma_{t}\left\langle-\sigma_{11}\right\rangle_{+}\right)\right]}{\gamma_{t}^{2}}
$$

Le comportement dans le sens des fibres est donc élastique non linéaire avec un module de traction $E_{1}^{T}$. Une approche similaire est classiquement développée pour les non-linéarités en compression.

$$
E_{1}^{T}=E_{1}^{0}\left(1+\gamma_{t}\left\langle\sigma_{11}\right\rangle_{+}\right) \quad E_{1}^{C}=E_{1}^{0}\left(1+\gamma_{c}\left\langle\sigma_{11}\right\rangle_{-}\right)
$$

Les trois forces thermodynamiques $Y_{d_{1}}, Y_{d_{2}}$ et $Y_{d_{12}}$, conjuguées des variables d'endommagement $d_{1}, d_{2}$ et $d_{12}$ et supposées uniformes dans l'épaisseur de la mono couche sont déterminées à partir de l'énergie de la monocouche.

$$
\begin{gathered}
Y_{d_{1}}=-\left.\frac{\partial E_{D}}{\partial d_{1}}\right|_{\sigma} \text { et } Y_{d_{2}}=-\left.\frac{\partial E_{D}}{\partial d_{2}}\right|_{\sigma}=\frac{\left\langle\sigma_{22}\right\rangle_{+}^{2}}{2 E_{2}^{0}\left(1-d_{2}\right)^{2}} \\
Y_{d_{12}}=-\left.\frac{\partial E_{D}}{\partial d_{12}}\right|_{\sigma}=\frac{\sigma_{12}^{2}}{2 G_{12}^{0}\left(1-d_{12}\right)^{2}}
\end{gathered}
$$

Les évolutions des deux variables d'endommagement $d_{2}$ et $d_{12}$ sont couplées car gouvernées par une force thermodynamique commune $\underline{Y}$ :

$$
\underline{\sqrt{Y}}=\sup _{t^{\prime} \leq t} \sqrt{Y_{d_{12}}+b Y_{d_{2}}}
$$

où $b$ est un paramètre de couplage du matériau. L'étude des résultats d'essais nous conduit classiquement à choisir l'évolution simplifiée des variables d'endommagement $d_{2}$ et $d_{12}$ (Ladevèze et Le Dantec, 1992) :

$$
d_{12}=\frac{\sqrt{Y}-\sqrt{Y_{120}}}{\sqrt{Y_{12 c}}} \text { et } d_{2}=\frac{\sqrt{Y}-\sqrt{Y_{20}}}{\sqrt{Y_{2 c}}}
$$

Les déformations anélastiques constatées lors des essais de traction sur des stratifications désorientées sont attribuées à des non refermetures de fissures et à des phénomènes de frottement entre la fibre et la matrice. Pour modéliser ce phénomène nous utilisons un modèle élastoplastique avec un écrouissage isotrope. Les déformations résiduelles dans les directions de la chaîne et de la trame doivent être prises en compte dans le cas des tissus. 


$$
f(\widetilde{\sigma}, R(p))=\sqrt{\left(\widetilde{\sigma}_{12}\right)^{2}+a_{1}^{2}\left(\widetilde{\sigma}_{11}\right)^{2}+a_{2}^{2}\left(\widetilde{\sigma}_{22}\right)^{2}}-R(p)-R_{0} \quad R(p)=\beta p^{\alpha}
$$

La déformation plastique cumulée est définie

$$
\dot{\widetilde{p}}=\left(4 \dot{\widetilde{\varepsilon}}_{12}^{p^{2}}+a_{1}{ }^{2} \dot{\widetilde{\mathcal{E}}}_{11} p^{2}+a_{2}{ }^{2} \dot{\widetilde{\varepsilon}}_{22}^{p^{2}}\right)^{1 / 2}
$$

La simulation du comportement en cisaillement est présenté en figure 2 pour le tissu équilibré HexForce 43199 200g/m2-époxy SR8100. Elle correspond aux résultats issus d'un essai de traction mené sur une stratification orienté à $45^{\circ}$ pour une vitesse de déformation quasi statique. Généralement les déformations irréversibles modélisées par la partie élastoplastique sont supposées négligeables dans le sens de la chaîne ou de la trame. Cependant, nous avons montré que dans le cas des tissus interlocks carbone époxy, elles peuvent être modélisées par le critère de plasticité proposé (Rozycki et Gornet, 2015).

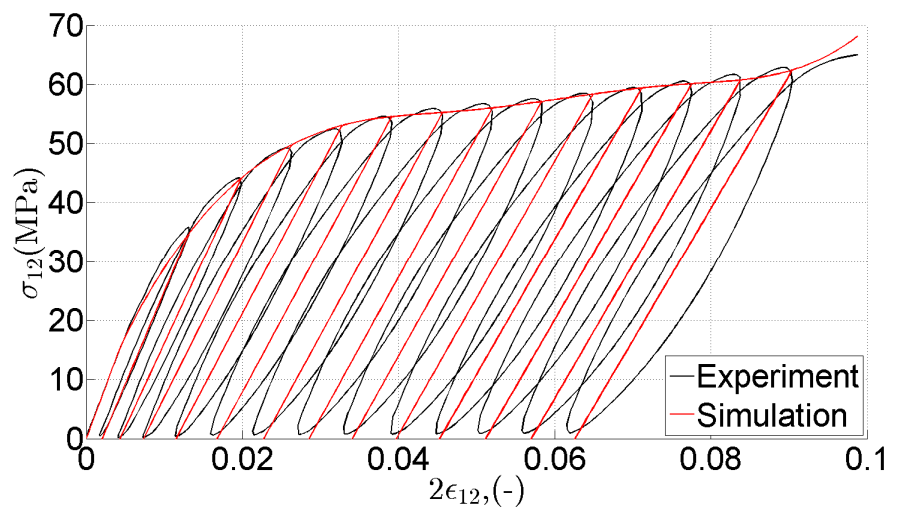

Figure 2. Réponse expérimentale en cisaillement plan et simulation élastoplastique endommageable. $\dot{\varepsilon}_{12}=0.05 \mathrm{~s}^{-1}$

Un modèle viscoélastique fractionnaire à déformations irréversibles pilotées par l'endommagement a été initialement proposé dans (Mateos et al. 2013). Nous montrons dans cette étude que les modèles de comportement élastoplastique endommageable (Ladevèze et Le Dantec, 1992 ; Hochard et al., 2011) peuvent être enrichis afin de prendre en compte les boucles d'hystérésis dues au cisaillement plan. Une dérivée fractionnaire de Riemann-Liouville est introduite afin de modéliser les boucles d'hystérésis. Dans le cadre des éléments finis, la régularisation de la rupture peut être réalisée par une approche non locale. Cette méthode permet de prendre en compte les effets d'échelle sur la rupture. La dérivée fractionnaire, pierre angulaire de notre modélisation s'écrit : 


$$
\begin{gathered}
D^{\alpha} \varepsilon_{12}(x)=\frac{1}{\Gamma(1-\alpha)} \frac{d}{d x} \int_{0}^{x}(x-t)^{-\alpha} \varepsilon_{12}(t) d t \text { avec } 0<\alpha<1 \\
\Gamma(z)=\int_{0}^{\infty} e^{-x} x^{z-1} d x, \quad z \in \mathbb{R}_{+}
\end{gathered}
$$

ou $D^{\alpha}$ représente la dérivée $\alpha$ de la déformation de cisaillement. La fonction Gamma généralise la définition des factoriels aux nombres non entiers. Il convient de noter que $\alpha$ évolue avec le niveau d'endommagement (figure 3).

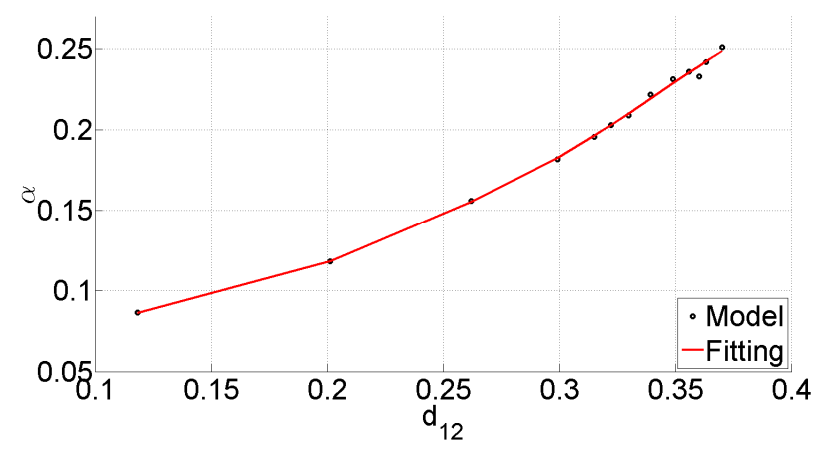

Figure 3. Évolution de l'exposant fractionnaire en fonction de l'endommagement

Le lien entre la viscosité et l'endommagement est un fait classiquement reconnu pour les matériaux composites. Afin d'améliorer la qualité des simulations une forme polynomiale est introduite pour l'évolution des endommagements. Le comportement en cisaillement pour un polynôme d'ordre quatre est présenté sur la figure 4.

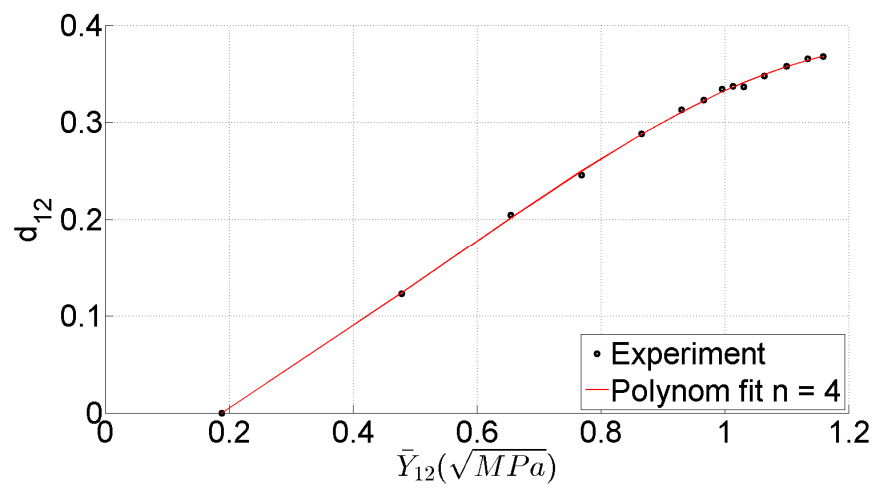

Figure 4. Endommagement en cisaillement plan. Identification d'un polynôme $d u 4^{e}$ ordre 


$$
d_{12}=\sum_{n=1}^{N} a_{n}\left(Y-Y_{120}\right)^{n} \quad d_{2}=\sum_{n=1}^{N} b_{n}\left(Y-Y_{20}\right)^{n}
$$

De même il est possible d'employer un écrouissage isotrope sous forme de puissance généralisée.

$$
R(p)=\sum_{\alpha=1}^{m} \beta_{\alpha} p^{\alpha}
$$

Lors de la charge, le critère de plasticité est activé ( $f=0$ et $\dot{f}=0)$. Dans la phase de décharge ou de recharge précédent le retour à la phase élastoplastique endommageable, le comportement visqueux fractionnaire est actif en cisaillement plan. Le comportement est assimilable à un modèle de fractionnaire de KelvinVoigt. La contrainte $\sigma_{12}^{P}\left(d_{12}\right)$ permet d'assurer la continuité en contrainte lors du passage du modèle élastoplastique endommageable au modèle fractionnaire. Le comportement en cisaillement plan avec boucles d'hystérésis s'écrit :

$$
\sigma_{12}=\sigma_{12}^{P}\left(d_{12}\right)+2 G_{12}^{v}\left(1-d_{12}\right) \frac{D^{\alpha} \varepsilon_{12}^{e}}{D t^{\alpha}}
$$

D'un point de vue numérique la dérivée fractionnaire est évaluée à l'aide de l'algorithme L1 proposé par (Grünwald 1867). La figure 5 illustre la réponse expérimentale en cisaillement plan et la simulation numérique avec prise en compte de l'hystérésis.

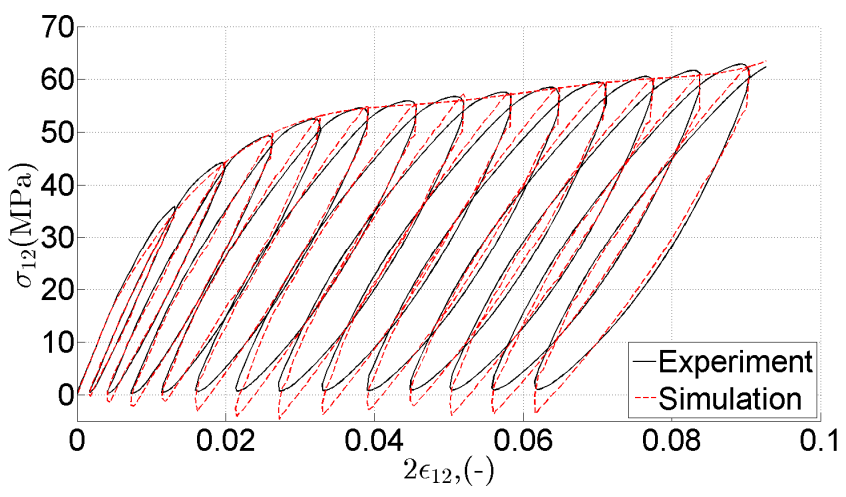

Figure 5. Réponse expérimentale en cisaillement plan et simulation numérique avec prise en compte de l'hystérésis. Les boucles sont correctement représentées par le modèle élastoplastique endommageable à dérivée fractionnaire. $\dot{\varepsilon}_{12}=0.05 \mathrm{~s}^{-1}$

La dissipation $\mathrm{du}$ modèle élastoplastique endommageable à dérivées fractionnaires du pli élémentaire de composite s'écrit : 


$$
\Phi=\sigma_{i j} \dot{\varepsilon}_{i j}^{p}+R \dot{p}+Y_{11} \dot{d}_{1}+Y_{22} \dot{d}_{22}+Y_{12} \dot{d}_{12}+2 \dot{\varepsilon}_{12}^{e} G_{12}^{v} \frac{D^{\alpha} \varepsilon_{12}^{e}}{D t^{\alpha}}
$$

Dans le cas d'un chargement cyclique l'énergie dissipée par l'hystérésis est prépondérante par rapport aux autres dissipations (Westphal 2014). L'évolution de la vitesse de sollicitation par rapport à une référence quasi-statique est également prise en compte dans le modèle à l'aide des formules 15 et 16 (Rozycki et Gornet 2015). Elle permet par exemple de simuler l'évolution du comportement en cisaillement (figure 6) en fonction de la vitesse de déformation.

$$
G_{12}=G_{12}^{0}\left[1+F_{G}\left(\dot{\varepsilon}_{12}^{e}\right)\right] \quad R_{0}=R_{0}^{0}\left[1+F_{R}\left(\dot{\varepsilon}_{12}^{e}\right)\right]
$$

$$
F_{G}=G^{*}\left(\frac{\left\langle\dot{\varepsilon}_{12}^{e}-\dot{\varepsilon}_{12}^{0}\right\rangle_{+}}{\dot{\varepsilon}_{12}^{0}}\right)^{m} \quad F_{G}=R^{*}\left(\frac{\left\langle\dot{\varepsilon}_{12}^{e}-\dot{\varepsilon}_{12}^{0}\right\rangle_{+}}{\dot{\varepsilon}_{12}^{0}}\right)^{k}
$$

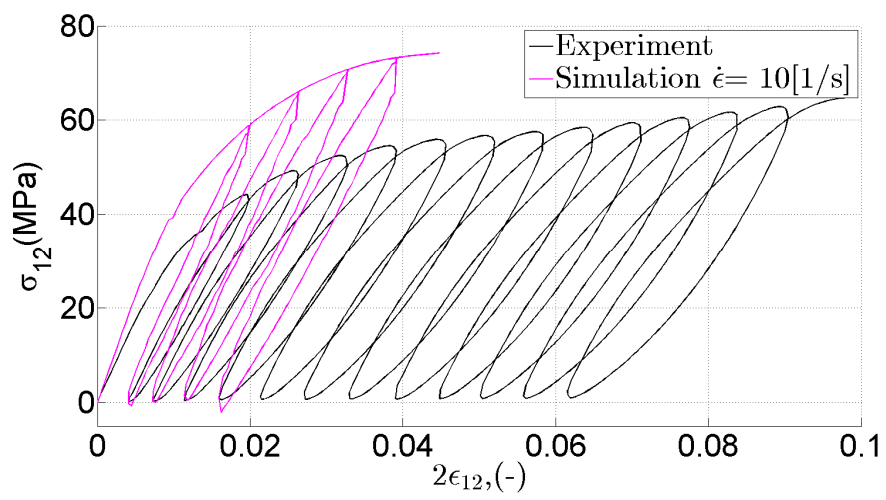

Figure 6. Réponse expérimentale en cisaillement plan et simulation numérique avec prise en compte de l'hystérésis et de l'effet de vitesse de chargement.

Vitesses de déformation $\dot{\varepsilon}_{12}^{0}=0.05 \mathrm{~s}^{-1}$ et $\dot{\varepsilon}_{12}=10 \mathrm{~s}^{-1}$

\section{Caractérisation mécanique d'un composite composé de plis unidirectionnels}

Les essais mécaniques destinés à la caractérisation des matériaux sont exécutés à l'aide d'une machine hydraulique MTS d'une capacité de 10 tonnes. Les éprouvettes de longueur utile $150 \mathrm{~mm}$ et de largeur constante $25 \mathrm{~mm}$ (normes ASTM D3039ISO527) sont équipées de talons orientés à $45^{\circ}$. L'épaisseur est fonction du nombre de couche et de leur grammage. Chaque éprouvette est instrumentée de deux rosettes bidirectionnelles juxtaposées disposées en vis-à-vis sur les faces de l'éprouvette afin 
de suivre l'évolution des déformations longitudinales et transversales au cours de l'essai. Il faut noter que l'essai de traction sur un composite multicouche stratifié impose en fait un chargement complexe au niveau du pli élémentaire unidirectionnel. Pour caractériser le pli élémentaire de matériau composite, des essais de traction ont été menés sur des empilements de type : $[0]_{8},[+45 /-45]_{2 s}$, $[+67.5 /-67.5]_{2 s}$. Ces trois séquences ont permis d'identifier les paramètres du modèle de la couche élémentaire. Des éprouvettes $[0 / 90]_{2 s}$ et $[0 / 45 /-45 / 90]_{2 s}$ ont également été utilisées pour les essais de fatigue. Enfin la résine époxy étant sensible à la vitesse de sollicitation, le comportement en cisaillement plan a été validé pour différentes vitesses. La thermographie passive est employée pour suivre en continu l'évolution du champ de température à la surface du matériau. Elle permet entre autres la localisation des sites d'initiation de l'endommagement. De plus, le suivi de la température permet de localiser la limite d'élasticité. La température décroît lors de la première phase de l'essai de traction. L'augmentation de la température est directement liée à la croissance des premiers endommagements. Des thermocouples $\mathrm{K}$ ou une caméra thermique de type Cedip Infrared Systems (matrice de 320*240 détecteurs Mercure-Cadmium-Telluride) complètent le dispositif de mesure. L'acquisition des données d'essais est réalisée grâce à une électronique de mesure Quantum HBM.

Il convient de noter que la réponse thermoélastique de l'éprouvette lors d'un essai de traction monotone permet de localiser la limite de fatigue du stratifié. Cette méthode est présentée pour les métaux dans (Risitano et al., 2011). A titre d'illustration, la figure 7 permet de localiser une limite de fatigue en cisaillement plan de $32 \mathrm{MPa}$ au point où la variation de température commence à croître. Cette valeur obtenue avec un simple essai de traction est cohérente avec celle déterminée par les essais d'auto-échauffement et de fatigue présentés dans la suite.

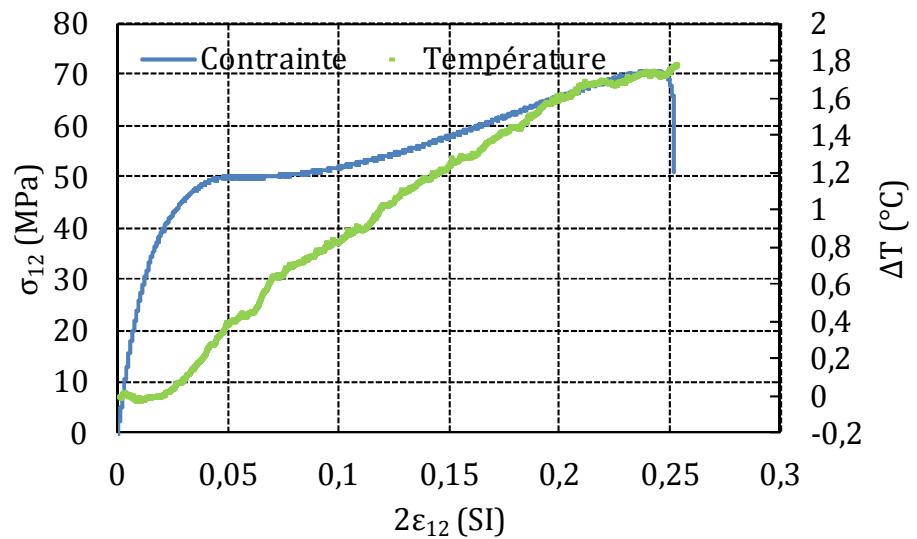

Figure 7. Évolution de la contrainte et de la température avec la déformation de cisaillement durant un essai de traction monotone sur $[+45 /-45]_{2 s}$

La contrainte de limite de fatigue en cisaillement correspond à la fin de la chute de température et est estimée à $32 \mathrm{MPa}$ 


\section{Caractérisation mécanique en fatigue à grands nombres de cycles}

Les essais d'auto-échauffement ont été menés pour des chargements cycliques de traction-traction à $\mathrm{R}=0,1$ ou à contrainte moyenne imposée. L'éprouvette subit une suite de plusieurs blocs de chargement à contrainte moyenne constante mais d'amplitude croissante. Durant chacun de ces blocs de chargement, on enregistre l'évolution de la température moyenne en surface d'éprouvette et on détermine une température moyenne stabilisée pour chaque bloc. Chaque bloc de chargement cyclique est précédé d'une montée en charge jusqu'à atteindre la contrainte moyenne choisie effectuée en contrôle en déplacement à une vitesse de $2 \mathrm{~mm} / \mathrm{min}$. La phase de chargement cyclique comprend 3000 cycles d'amplitude de contrainte constante à une fréquence de $5 \mathrm{~Hz}$, de façon à ce que l'éprouvette stratifiée atteigne un état d'équilibre thermique. Ensuite, un retour à contrainte nulle à une vitesse de déplacement de la traverse de $2 \mathrm{~mm} / \mathrm{min}$ est réalisé. Entre chaque bloc de chargement, des temporisations sont introduites afin de permettre un retour de l'équilibre thermique de l'éprouvette à la température ambiante (figure 8). La température en surface des éprouvettes durant les chargements cycliques est mesurée à l'aide d'une caméra thermique Cedip. Le suivi de la température a également été validé avec des thermocouples $\mathrm{K}$ afin de rendre la méthode utilisable avec un environnement industriel.

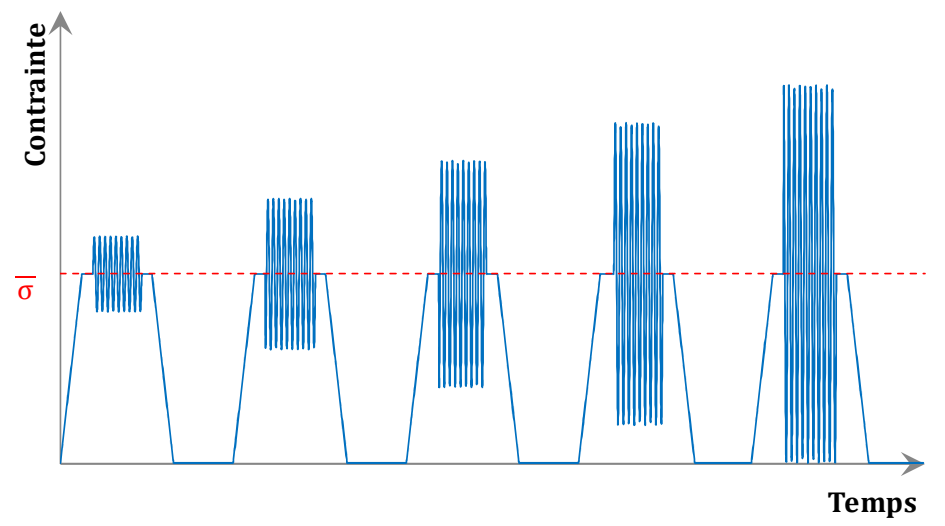

Figure 8. Déroulement d'un essai d'auto-échauffement à contrainte moyenne imposée. Blocs de chargements cycliques imposés à l'éprouvette pour un essai à contrainte moyenne imposée

\subsection{Méthode d'auto-échauffement, mesures thermiques}

La température moyenne en surface d'éprouvette a été déterminée en moyennant les valeurs de chaque pixel de la caméra thermique inclus dans une fenêtre de mesure, correspondant à la partie utile de l'éprouvette. L'émissivité de la surface des échantillons a été déterminée expérimentalement en comparant les résultats de 
thermocouples et les données recueillies par la caméra thermique. Étant donné la faible épaisseur des éprouvettes employées, on suppose que le gradient de température dans l'épaisseur est négligeable. A partir d'une image enregistrée par la caméra à l'instant $t$, on relève la température moyenne à la surface de la zone utile de l'éprouvette $T(t)$, la température du mors haut $T_{h}(t)$ et la température du mors bas $T_{b}(t)$. La variation de température moyenne $\theta(t)$ est calculée à partir des valeurs relevées pour chaque pixel compris dans la zone utile de l'éprouvette. Ces trois mesures permettent de calculer les variations de température moyenne du stratifié par l'équation (17):

$$
\theta(t)=T(t)-T(0)-\frac{T_{h}(t)-T_{h}(0)+T_{b}(t)-T_{b}(0)}{2}
$$

$T(0), T_{h}(0)$ et $T_{b}(0)$ correspondent respectivement à la température moyenne initiale en surface de l'éprouvette, la température initiale du mors haut et la température initiale du mors bas. Cette méthode permet de s'affranchir de l'élévation de température due à l'augmentation de la température de l'huile dans la chambre du vérin.

\subsection{Limite de fatigue par la méthode d'auto-échauffement et courbes de Wöhler}

Dans cette partie, on étudie le domaine de fatigue d'un pli unidirectionnel de carbone époxy TR50-R367-2 autour d'une contrainte apparente moyenne située dans le domaine élastique. Le niveau de chargement moyen macroscopique de la stratification $[+45 /-45]_{2 \mathrm{~s}}$ a été choisi égal au tiers de la contrainte à rupture. Cette valeur correspond à un peu plus que la moitié de la contrainte élastique de cisaillement plan. Les niveaux des contraintes apparentes des éprouvettes stratifiées $[+67.5 /-67.5]_{2 \mathrm{~s}}$, et $[45 /-45 / 90 / 0]_{\mathrm{S}}$ ont été déterminés de façon à solliciter les plis élémentaires à un niveau identique de contrainte. Le chargement mécanique dans le pli à $45^{\circ}$ de la stratification quasi-isotrope est identique à celui du pli dans la stratification $[+45 /-45]_{2 \mathrm{~s}}$. De la même façon, le chargement moyen dans le pli à $90^{\circ}$ de la stratification quasi-isotrope est identique à celui subi par le pli à $90^{\circ}$ dans la stratification croisée $[0 / 90 / 0 / 90]_{\mathrm{S}}(c f$. tableau 1$)$. Les chargements étudiés sont destinés à déterminer les limites de fatigue dans les directions du pli élémentaire gouvernées par le comportement de la matrice. Les courbes d'auto-échauffement (figures 9, 10-12) représentent l'évolution de la température moyenne stabilisée de la surface de l'éprouvette en fonction de la contrainte maximale appliquée. Un point sur la courbe correspond à un bloc de chargement d'amplitude donné. La stabilisation de la température moyenne signifie que la dissipation thermique est constante. Il existe donc un état stationnaire de température correspondant à un équilibre thermique entre la quantité de chaleur perdue par conduction, convection, rayonnement et celle crée par les dissipations dues aux mécanismes de viscosité de la matrice et de sa dégradation. 


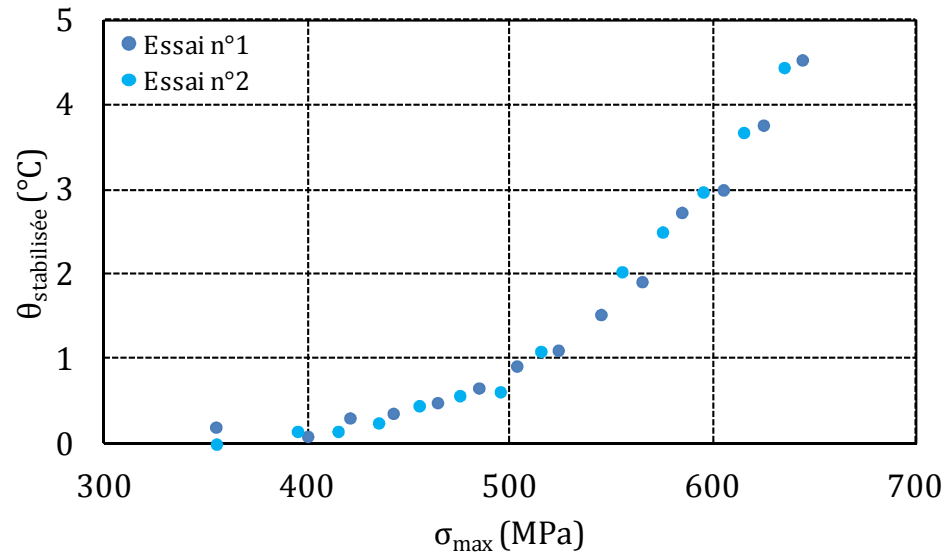

Figure 9. Réponses thermiques de deux essais d'auto-échauffement sur une stratification $\left[(0 / 90)_{2}\right]_{S}$ testée sous une contrainte macroscopique moyenne constante de $335 \mathrm{MPa}$

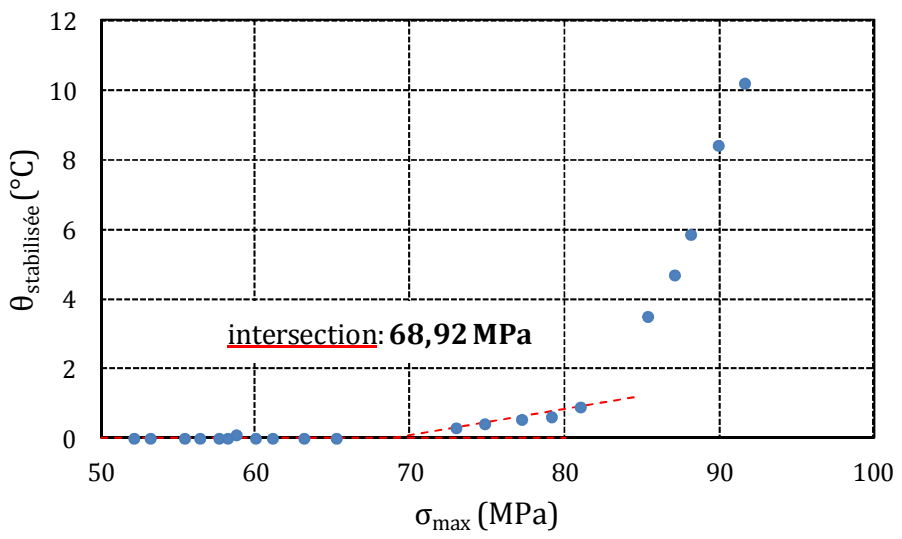

Figure 10. Dépouillement empirique de la courbe d'auto-échauffement obtenue pour la stratification $\left[( \pm 45)_{2}\right]_{s}$ sous un chargement à contrainte moyenne constante

L'intersection de la droite avec l'axe des abscisses fournit la limite d'endurance des stratifications sous les conditions de chargement testées. Cette hypothèse est corroborée par des essais classiques de fatigue (figures 11 et 13). On peut expliquer les ruptures de pente de la courbe d'auto-échauffement par l'activation de nouveaux mécanismes dissipatifs, qui viennent accroître l'énergie dissipée et donc la chaleur dégagée par l'éprouvette. L'essai d'auto-échauffement mené sur l'éprouvette quasi- 
isotrope [45/-45/90/0]s a permis de retrouver directement à partir d'un seul essai les limites de fatigue des deux stratifications avec un seul essai en considérant l'éprouvette à la limite de son domaine élastique. Les chargements imposés aux éprouvettes d'auto échauffement ainsi que et la synthèse des limites de fatigue sont présentés dans le tableau 1.

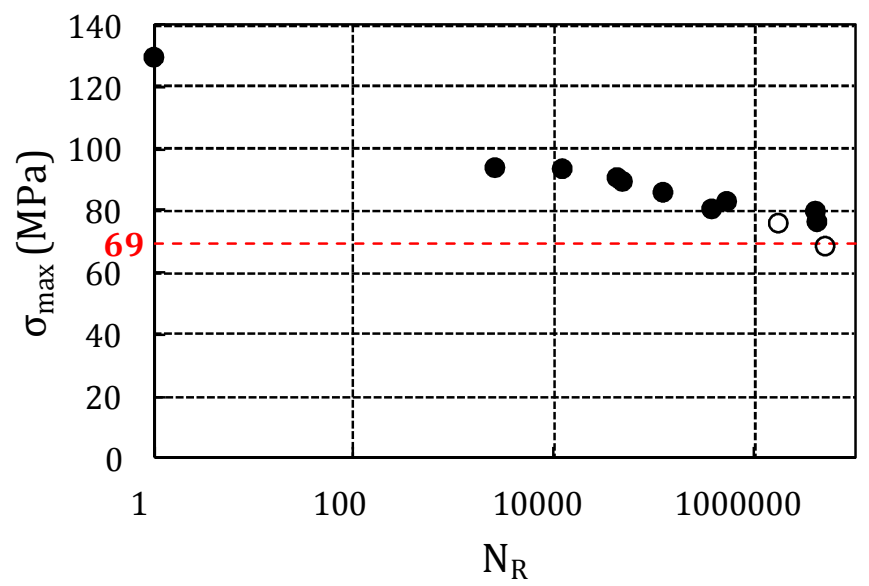

Figure 11. Courbe S-N. Confrontation entre les essais de fatigue classique et la limite d'endurance fournie par la méthode de dépouillement de la courbe d'auto-échauffement proposée pour la stratification $\left[( \pm 45)_{2}\right]_{S}$

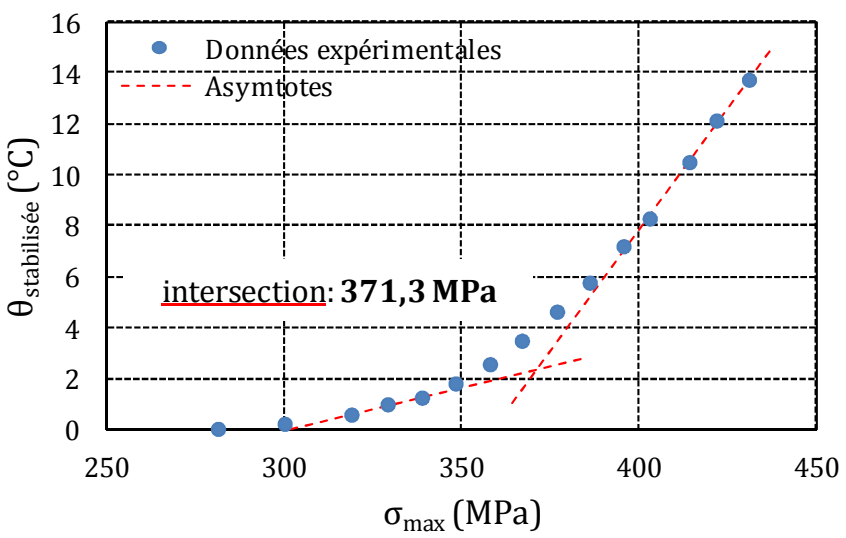

Figure 12. Dépouillement empirique de la courbe d'auto-échauffement obtenue pour la stratification quasi-isotrope $[ \pm 45 / 90 / 0]_{S}$ sous une contrainte moyenne $\bar{\sigma}_{x x}=250 \mathrm{MPa}$ 


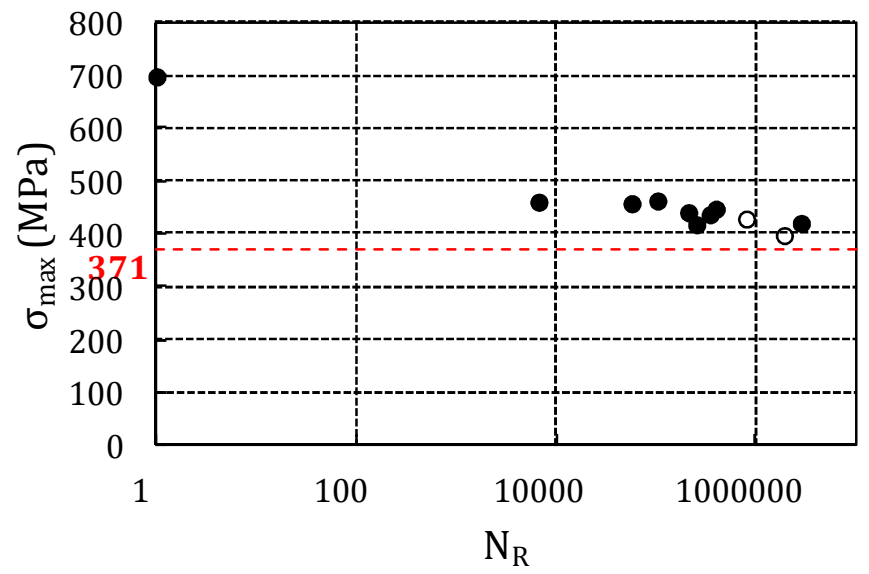

- Fatigue classique (éprouvettes rompues)

- Fatigue classique (éprouvettes non rompues)

-.-.- Auto-échauffement

Figure 13. Courbe S-N. Confrontation entre les essais de fatigue classique et la limite d'endurance fournie par la méthode de dépouillement de la courbe d'auto-échauffement proposée pour la stratification $[ \pm 45 / 90 / 0]_{S}$

Tableau 1. Chargement des éprouvettes stratifiées à contrainte moyenne imposée et limites de fatigue déterminées à partir des essais d'auto-échauffement

\begin{tabular}{|c|c|c|c|}
\hline $\begin{array}{c}\text { Stratifications } \\
\text { testées }\end{array}$ & $\begin{array}{c}\text { Chargement } \\
\text { Contrainte imposée }\end{array}$ & $\begin{array}{c}\text { Contraintes } \\
\text { imposées } \\
\text { Échelle du pli }\end{array}$ & Limite de fatigue \\
\hline$\left[( \pm 45)_{2}\right]_{s}$ & $\bar{\sigma}_{x x}=50 \mathrm{MPa}$ & $\sigma_{12}=25 \mathrm{MPa}$ & $\sigma_{12}=34,5 \mathrm{MPa}$ \\
\hline$\left[(0 / 90)_{2}\right]_{S}$ & $\bar{\sigma}_{x x}=335 \mathrm{MPa}$ & $\begin{array}{c}\text { Pli à } 90^{\circ}: \\
\sigma_{22}=41 \mathrm{MPa}\end{array}$ & $\begin{array}{c}\text { Pli à } 90^{\circ}: \\
\sigma_{22}=71 \mathrm{MPa}\end{array}$ \\
\hline$[ \pm 45 / 90 / 0]_{s}$ & $\bar{\sigma}_{x x}=250 \mathrm{MPa}$ & $\begin{array}{c}\sigma_{12}=25 \mathrm{MPa} \\
\text { Pli à } 95^{\circ}:\end{array}$ & $\begin{array}{c}\text { Pli à } 45^{\circ}: \\
\sigma_{12}=32 \mathrm{MPa} \\
\text { Pli à } 90^{\circ}:\end{array}$ \\
& & $\sigma_{22}=42 \mathrm{MPa}$ & $\sigma_{22}=69 \mathrm{MPa}$ \\
\hline
\end{tabular}

En conclusion, il suffit de réaliser pour chaque stratification, un essai à rupture quasi-statique permettant de localiser la limite de fatigue, quelques essais de fatigue à forts niveaux de contraintes et enfin des essais d'auto échauffement pour localiser 
précisément l'asymptote de la limite de fatigue. Les courbes de Wöhler S-N sont modélisables par :

$$
\sigma_{12}=\sigma_{12 S}-B_{12} \log \left(N_{i}\right) \text { et } \sigma_{22}=\sigma_{22 S}-B_{22} \log \left(N_{i}\right)
$$

En conséquence, quatre essais par stratification semblent suffisants pour identifier et valider la modélisation des courbes $\mathrm{S}-\mathrm{N}$ par deux droites. La deuxième droite correspondant à une droite horizontale précisant la limite de fatigue. La répétabilité des essais de fatigue est nécessaire pour valider l'aspect probabiliste de la rupture. Il convient de rappeler que l'essai d'auto-échauffement n'est pas sensible aux imperfections des matériaux contrairement aux essais traditionnels de fatigue. En fatigue traditionnelle une imperfection dans la stratification peut conduire à une rupture prématurée de l'éprouvette.

\subsection{Simulations éléments finis des essais d'auto-échauffement}

Des simulations numériques ont également été effectuées avec le code éléments finis Cast3M dans le but de retrouver les valeurs de température moyenne stabilisée pour les différentes stratifications étudiées en auto-échauffement. L'équation de la chaleur a été résolue en régime stationnaire (figures 14 et 15) et instationnaire. Ces simulations nécessitent la détermination des propriétés de conduction du pli élémentaire (Westphal, 2014). La source de dissipation $\Phi$ a été imposée dans le modèle éléments finis thermique de plaque stratifié (figure 14).

La dissipation d'énergie expérimentale a été identifiée pour chaque bloc de chargement de 3000 cycles. Les dissipations sont dues à la plasticité, à l'endommagement et à la viscosité du pli élémentaire de carbone/époxy (aire de l'hystérésis de la courbe contrainte-déformation). Les températures atteintes lors des essais d'auto-échauffement sont simulées par éléments finis avec succès. Du point de vue de la simulation, la dissipation thermique déterminée expérimentalement est introduite dans le modèle éléments finis de thermique stratifié à l'aide d'une source de chaleur volumique. La plus grande variation de température atteint $12{ }^{\circ} \mathrm{C}\left(35^{\circ} \mathrm{C}\right.$ $23{ }^{\circ} \mathrm{C}$ ) pour les éprouvettes $[ \pm 45 / 90 / 0]_{\mathrm{S}}$ sollicitées sur 3000 cycles. Dans le cas des éprouvettes $[45 /-45]_{4 \mathrm{~S}}$ la variation maximale de température est de $22{ }^{\circ} \mathrm{C}$. Les essais mécaniques sont réalisés sur une MTS hydraulique. En conséquence la température n'est pas symétrique car le mors est plus chaud du côté du vérin hydraulique (figure 14). Un palier d'auto-échauffement peut être modélisé par un état de thermique stationnaire. Les conditions aux limites du problème de thermique stationnaire de la plaque stratifiée sont les températures imposées des mors bas et haut, la convection naturelle sur les deux faces de l'éprouvette et la source volumique de dissipation. Il convient de rappeler que dans une approche simplifiée le rayonnement est intégré au coefficient de convection naturelle. Cette méthode permet de conduire des simulations linéaires simplifiées. Le coefficient de convection corrigé du rayonnement est recalé sur le bloc de cycles ou la variation de température $\theta$ est la plus grande. Cette méthode conduit à une erreur de $8 \%$ sur les blocs de faible variation de température $\left(1\right.$, à $\left.2{ }^{\circ} \mathrm{C}\right)$. Les simulations éléments finis 
de thermiques linéaires sont satisfaisantes pour déterminer le champ de températures des éprouvettes. La simulation thermique de l'éprouvette quasi-isotrope $[ \pm 45 / 90 / 0]_{\mathrm{S}}$ est présentée sur la figure 14. Les simulations permettent de mettre en évidence l'importance des conditions de convexion sur la température de l'éprouvette pendant l'essai d'auto-échauffement. Ces constations avaient également été observées d'un point de vue expérimental.
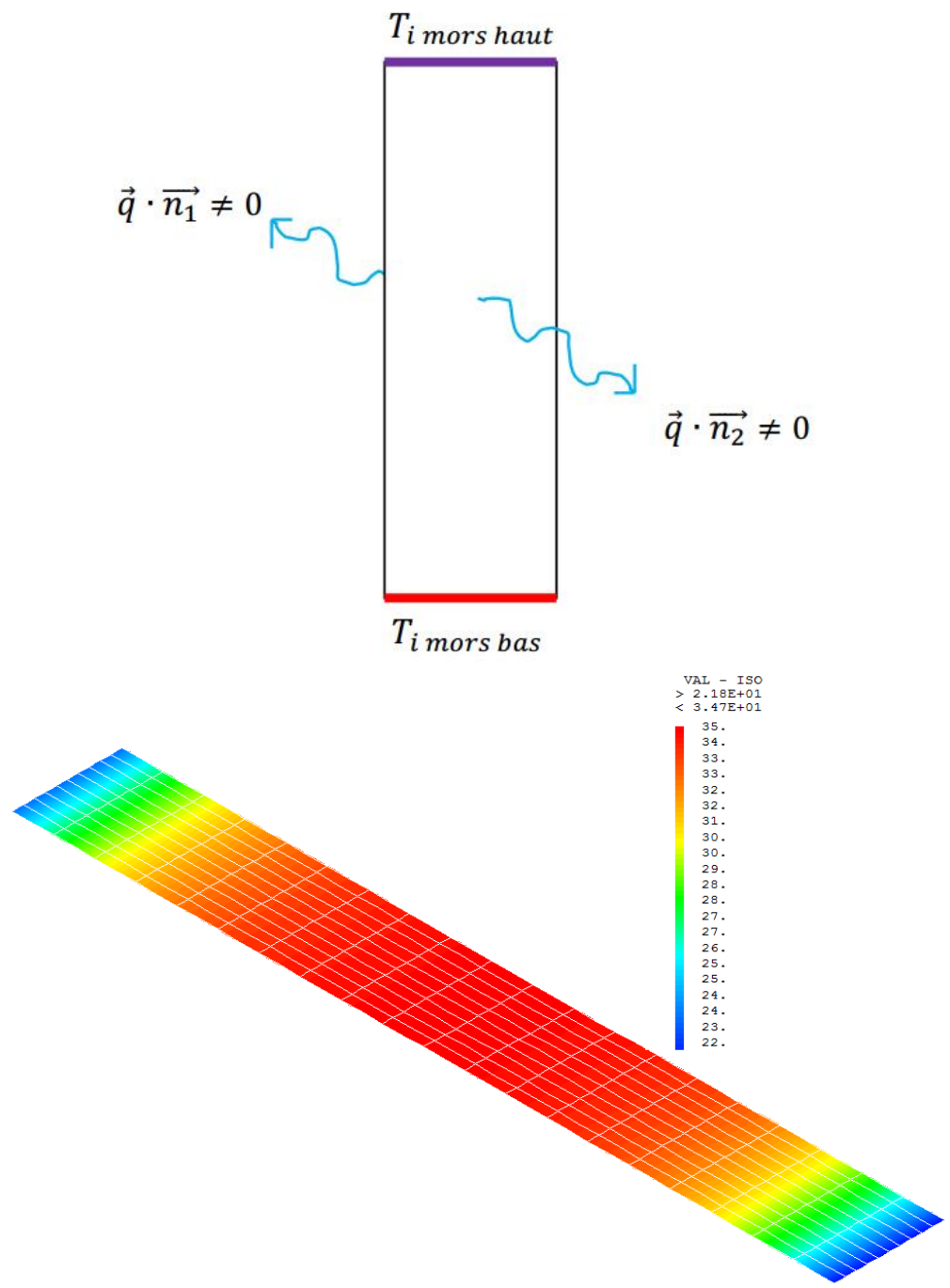

Figure 14. Simulation éléments finis en thermique stationnaire d'une éprouvette [ $\pm 45 / 90 / 0]_{s}$ pour le chargement mécanique maximum d'auto-échauffement. La source de dissipation $\Phi$ la convection naturelle et les températures des mors sont imposées. La température maximum atteinte par la peau est de $35^{\circ} \mathrm{C}$. 


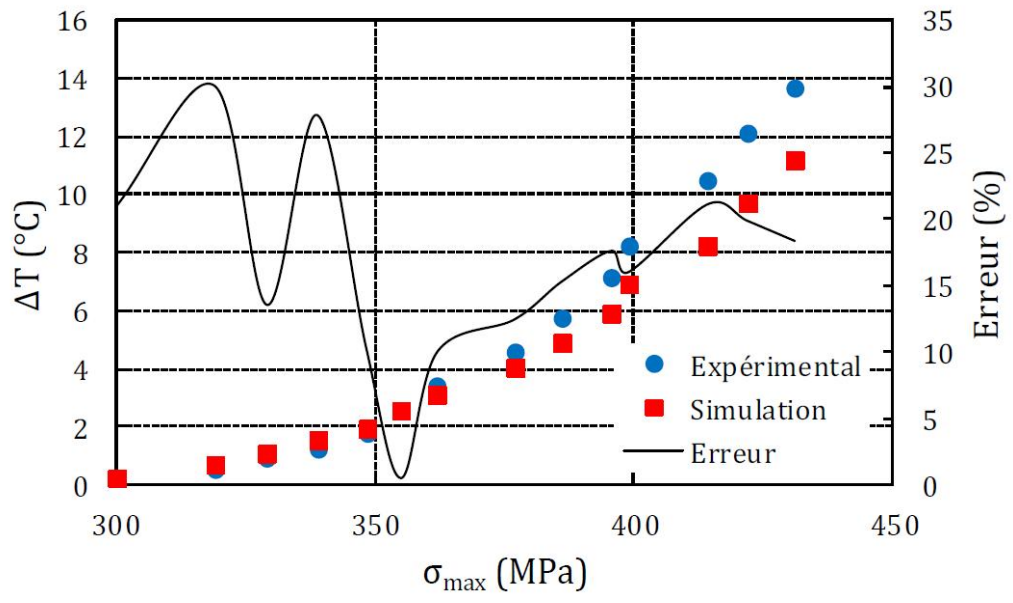

Figure 15. Éprouvette $[ \pm 45 / 90 / 0]_{s}$. Simulation de l'évolution de la variation de température par rapport à la température initiale de l'éprouvette en fonction de la contrainte maximum appliquée $\sigma_{x x M a x}$ pendant 3000 cycles

\section{Conclusion}

La simulation précise des endommagements et des échauffements du pli élémentaire constituant un composite stratifié carbone époxy peut être réalisée à l'aide d'un modèle élastoplastique endommageable à dérivée fractionnaire. Le modèle permet de simuler les différentes sources de chaleur dégagées lors de la sollicitation mécanique du composite. Dans cette étude, nous avons mis en évidence l'intérêt des mesures expérimentales d'auto-échauffement pour l'analyse du comportement en fatigue des stratifiés. Il convient de rappeler que lors d'un essai de traction monotone le point d'inversion des mécanismes thermoélastiques permet de localiser la limite de fatigue de l'éprouvette. L'analyse des courbes expérimentales d'auto-échauffement permet de déterminer les contraintes limites de fatigue du pli élémentaire de carbone époxy de façon fiable en seulement quelques heures. Ces valeurs s'avèrent cohérentes avec celles fournies par des essais de fatigue traditionnels. Pour le carbone époxy, l'emballement de la température est lié à la naissance et au frottement des lèvres des fissures dans les plis. L'étude tomographique des éprouvettes permet de montrer que les fissures issues des essais d'auto-échauffement sont comparables à celles obtenues par des essais de fatigue. L'analyse tomographique renforce notre conviction qu'il est pertinent d'utiliser la méthode d'auto-échauffement pour déterminer les limites en fatigue à grands nombre de cycles des composites. 


\section{Bibliographie}

Chrysochoss A., Louche H. (2000). An infrared image processing to analyse the calorific effect acccompagnying strain localization. International journal of engineering science, 38 (16), 1759-1788.

Doudar C., Calloch S., Hild F., Cugy P., Galtier A. (2004). Identification of the scatter in high cycle fatigue from temperature measurements. Comptes Rendus Mécanique, 332,795-801.

Grünwald (1867). Uber begrenzte Derivationen und deren Anwendung Z. Math. Und Phys. $12,441-480$.

Gornet L., Ijaz H. (2011). A high cyclic elastic fatigue damage model for carbon fiber epoxy matrix laminates with different mode mixtures. Journal Composites Part B: Engineering, 42, 1173-1180.

Ijaz H., Asad M., Gornet L., Alam Y.(2014). Prediction of delamination crack growth in carbon fiber epoxy composite laminates using non local damage model. Mchanics \& industry, 15 (4), 293-300.

Gornet L., Marckmann G., Ollier G. (2006). Interaction modèles expériences sur les âmes nids d'abeilles Nomex. Revue des composites et des matériaux avancés, vol. 16, 167-190.

Hochard C., Aubourg P.A., Charles J.P. (2011). Modelling of the mechanical behavior of woven-fabric CFRP laminates up to failure. Composites Science and Technology, 61, 2001, 221-230.

Luong MP, Dang Van K. (1992). Infrared thermographic evaluation of fatigue limit in metals. 27th QIRT Eurotherm Seminar, Paris France.

La Rosa G., Risitano A. (2000). Thermographic methodology for rapid determination of the fatigue limit of materials and mechanical components. International Journal of Fatigue, vol. 22, 65-73.

Ladevèze P., Le Dantec E. (1992). Damage modelling of the elementary ply for laminated composites. Composites Science and Technology, 43-3, 257-268.

Ladevèze P., Allix O., Gornet L., Leveque D. et Perret L. (1998). A Computational Damage Mechanics Approach for Laminates : Identification and Comparison with Experimental Result. Damage Mechanics in Engineering Materials, Studies in Applied Mechanics 46, G.Z. Voyiadjis, J.W. Ju \& J.-L. Chaboche eds. Elsevier, 481-500.

Mateos M., Gornet L., Rozycki P. (2013). Hysteretic behaviour of fibre reinforced composites under shear. Journée Nationale des Composites 18, Nantes.

Marguet P., Rozycki, Gornet L. (2007). A rate dependent constitutive model for carbon-fiber reinforced plastic woven fabrics. Journal Mechanics of Advanced Materials and Structures, 14, 2007, 619-631.

Naderi M., Kahirdeh A., Khonsari M. (2012). Dissipated thermal energy and damage evolution of Glass/Epoxy using infrared thermography and acoustic emission. Composites: Part B, vol. 43, 1613-1620.

Oldham K.B., Spanier J. (1974). The fractional calculus. Academic Press, New York and London.

Risitano A., Clienti C., Risitano G. (2011). Determination of fatigue limit by mono axial tensile specimens using thermal analysis. Key Engineering Materials 452, 361-364. 
Rozycki P., Gornet L. (2015). A general behaviour law in order to model thermoset and thermoplastic composite materials subjeted to dynamics loads. Copenhagen, ICCM 2015.

Westphal O. (2014). Analyse thermomécanique de l'endommagement de stratifiés carbone époxy: détermination de la limite d'endurance à partir d'essais d'auto-échauffement. Thèse, Ecole Centrale Nantes. 